\begin{tabular}{|c|c|c|c|c|c|}
\hline MUNIBE Antropologia-Arkeologia & $n^{\circ} 71$ & $91-102$ & DONOSTIA & 2020 & ISSN 1132-2217 • eISSN 2172-4555 \\
\hline
\end{tabular}

\title{
Pulidores de piedra pómez en la costa sur del Golfo de Bizkaia (España)
}

\author{
Pumice stone polishers on the southern coast \\ of the Bay of Biscay (Spain)
}

PALABRAS CLAVES: Roca volcánica. Huellas de uso. Recursos marinos. Magdaleniense superior. Funcionalidad. GAKO-HITZAK: Sumendi-arroka. Erabilera-arrastoa. Itsas baliabideak. Goi Madelein aldia. Funtzionaltasuna. KEY WORDS: Volcanic rock. Traces of use. Marine resources. Late Magdalenian. Functionality.

José Luis ARRIBAS PASTOR(1), Eduardo BERGANZA GOCHI(2)

\section{RESUMEN}

Se han estudiado tres pulidores de piedra pómez con ranuras de características formales y huellas de uso muy similares. Dos han sido hallados en el nivel III del yacimiento de la cueva de Santa Catalina (Lekeitio, Bizkaia); este nivel ha proporcionado un abundante y variado conjunto tecnoindustrial del Magdaleniense superior, datado en el Tardiglaciar. El tercero procede de un nivel Magdaleniense de la cueva de Aitzbitarte IV (Errenteria, Gipuzkoa). La función de estos objetos está relacionada con los procesos de fabricación de agujas o apuntados en hueso, asta u otras materias como la madera. Pulidores semejantes del mismo período cultural, también en piedra pómez, se han localizado en un territorio relativamente limitado, en el suroeste de Francia, junto a la Cordillera pirenaica. El área de localización de todos estos útiles era cercana a la costa, ecosistema en el que sería recogida la piedra pómez arrastrada por las corrientes marinas hasta el litoral del Golfo de Bizkaia, del que se difundiría hacia zonas interiores.

\section{LABURPENA}

Apar-harrizko hiru leungailu ikertu dira eta berdintsuak dira artekak eta erabilera-arrastoak hiruretan. Bi, Santa Catalina (Lekeitio, Bizkaia) aztarnategiko III. mailan aurkitu dira. Maila horretan, Tardiglaziarrean datatutako Goi Madeleine aldiko pieza ugari eta anizkoitzez osaturiko multzo teknoindustriala lortu da. Hirugarrena, Aitzbitarte IV (Errenteria, Gipuzkoa) aztarnategiko Madalen aldiko mailan topatu da. Objektu hauek orratzak edo hezurrezko zein adarrezko zein egurrezko puntadunak egiteko erabili ziren. Garai bereko antzeko leungailuak, apar-harrizkoak baita, inguru nahiko mugatuan, Frantziako hego-mendebaldean, Piriniotar mendikatearen parean aurkitu dira. Tresna hauek itsasbazterraren inguruan zabalduta egon dira; ekosistema horretan bilduko zen itsas korronteak Bizkaiko golkoko itsasertzeraino ekarritako apar-harria; handik, barrualderantz hedatuko zen.

\section{ABSTRACT}

Three fragments of pumice stone have been recovered at two sites near the southern coast of the Bay of Biscay, with extensive grooves and traces of wear caused by their use as polishers. Two come from level III of the Santa Catalina cave, dating from between 12900 and 15000 Cal $\mathrm{BP}$, and one from level IV of Aitzbitarte IV, with no radiocarbon dating. All three were found in Magdalenian cultural contexts.

The most plausible hypothesis concerning their acquisition is that they were picked up on the beaches near the settlements. The Santa Catalina cave, now open on a cliff, was about $5 \mathrm{~km}$ from the coast at that time. The archaeological record from level III of Santa Catalina attests to the fact that its occupants fished extensively and collected marine resources during the final phases of the Late Glacial. The Aitzbitarte IV cave was not far from the sea either.

A small number of similar tools were found on the northern slope of the Pyrenees, which have also been attributed to the same cultural period. With the exception of one found in a cave in the central Pyrenees, they all lie in a small area of south-western France, in settlements close to each other. These pumice stones must have had a similar origin to the specimens found in the cavities along the Cantabrian coast: volcanic areas in the Arctic or the Caribbean, from where they would have floated, carried by the sea currents. It is very possible that it was obtained by being collected on the Atlantic paleo-coast, although it cannot be ruled out that, in some cases, it was due to exchanges between neighbouring communities.

These types of tools have been linked to working with hard animal materials, in particular with making needles or other pointed objects, although we should not rule out other possible uses.

\footnotetext{
(1) AOZTA. jarribas@irakasle.eus

(2) AOZTA. eduardoberganza@irakasle.eus
} 


\section{INTRODUCCIÓN}

En algunos yacimientos de finales del Paleolítico superior de la zona atlántica del continente europeo no es infrecuente encontrar rocas con hendiduras o desgastes en la superficie de sus caras producidas por un rozamiento funcional con otra materia que se denominan pulidores con ranuras. Un reducido número de ellos son fragmentos de piedra pómez (pumita), roca ígnea, vítrea, de origen eruptivo de baja densidad debido a su estructura porosa.

En el nivel III del yacimiento de la cueva de Santa Catalina (Lekeitio, Bizkaia), en el que se ha determinado una sucesión de ocupaciones de grupos de cazadores-pescadores-recolectores con un equipamiento industrial lítico y óseo propio del Magdaleniense superior, datadas entre el 12.900 y el 15.000 Cal BP, se han recuperado dos fragmentos de piedra pómez de esas características. El registro arqueológico de industria lítica, ósea y de piezas de arte mueble es muy abundante y variado en este nivel. Destaca, además, por la profusión y singularidad de los restos paleontológicos, en especial los que tienen su origen o pueden ponerse en relación con un ecosistema marino y con la explotación de los recursos que se pueden obtener del mismo (Berganza et al. 2012; Berganza y Arribas, 2014; Castaños, 2014; Roselló-Izquierdo et al. 2015; Pétillon, 2016).

Presentamos en este artículo el análisis de las características morfológicas de esos objetos y de un tercero similar procedente de la cueva de Aitzbitarte IV (Errenteria, Gipuzkoa). Así mismo, esbozamos una aproximación a su posible función dentro de las cadenas operativas de fabricación de utillaje en las que pudieron intervenir. Finalmente, los hemos puesto en relación con objetos semejantes procedentes de yacimientos de un entorno geográfico próximo al de los estudiados. La metodología aplicada ha incluido el análisis microscópico a través de una lupa binocular
Olympus SZX10 con el fin de certificar su petrología. Este recurso también ha servido para examinar y describir las improntas generadas durante su uso como instrumento de trabajo.

\section{ANÁLISIS DE LOS PULIDORES}

\subsection{Cueva de Santa Catalina}

Pulidor $n^{\circ} 1$ (SC.A8.122.33)

Pieza de forma cónica con bordes y aristas redondeados; la base de forma subcuadrangular es plana y el extremo superior o distal apuntado, de sección oval con el ápice romo. Presenta una estructura con abundantes vesículas. Tiene color grisáceo, con tonos amarillentos, y es de reducidas dimensiones: $31,8 \mathrm{~mm}$ de altura y $37,4 \times 28.8 \mathrm{~mm}$ de anchura en la base. El peso es de 8,77 gr. Su actual estado de conservación puede considerarse bueno.

El exterior presenta tres caras claramente diferenciadas, todas de superficie convexa. En la cara $A$ se aprecian tres surcos profundos, paralelos entre sí y de orientación ligeramente oblicua con respecto al eje vertical. Nacen en el borde inferior y se prolongan hacia el extremo superior, sin llegar a alcanzarlo. Los dos surcos de la izquierda son más largos (27 mm) que el situado a su derecha $(17 \mathrm{~mm})$. Los tres tienen una morfología similar con el extremo inferior ancho y el superior apuntado. La sección de los de la izquierda es en $\mathrm{U}$ y la del tercero en $\mathrm{V}$, todos ellos con bordes rectos y paralelos. La profundidad es igual en todos ellos $(0,2$ $\mathrm{mm}$ ) (figura 1).

A la izquierda de las hendiduras anteriores hay una zona desgastada de unos $7 \mathrm{~mm}$ de anchura, que marca una somera depresión, paralela al surco de la izquierda, sin que se haya llegado a producir una acanaladura apreciable (Figura 2).
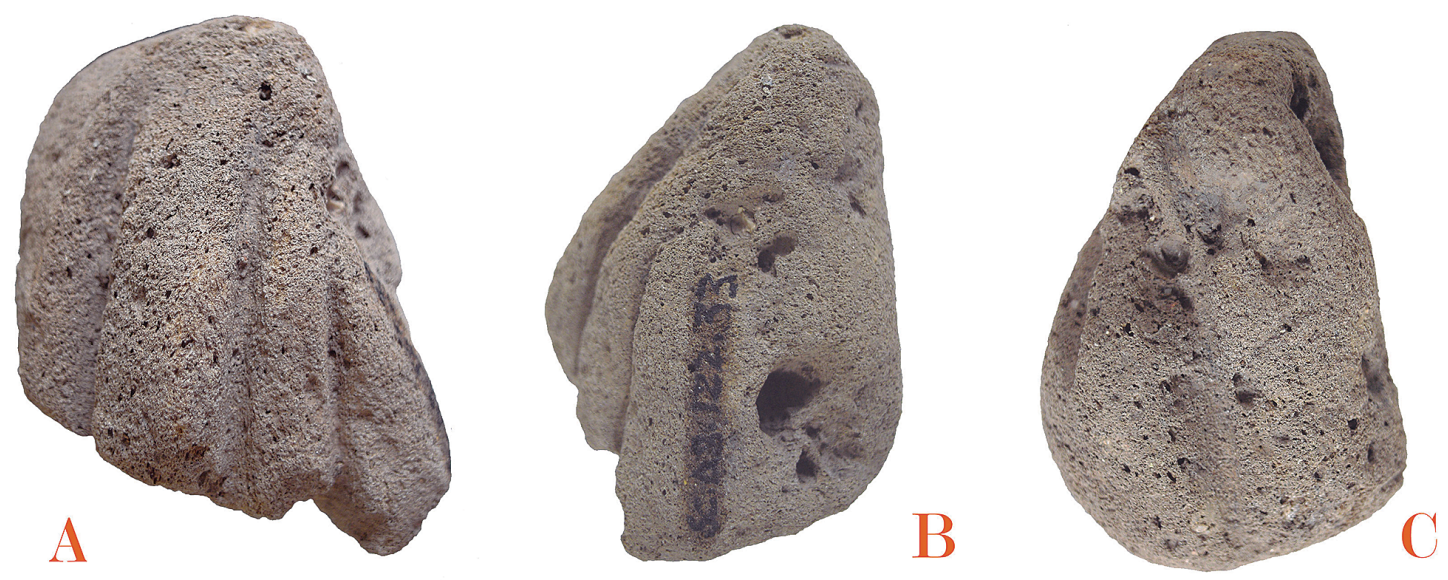

Fig.1. Pulidor $n^{\circ}$ 1 de Santa Catalina. / Polisher No. 1 from Santa Catalina. 


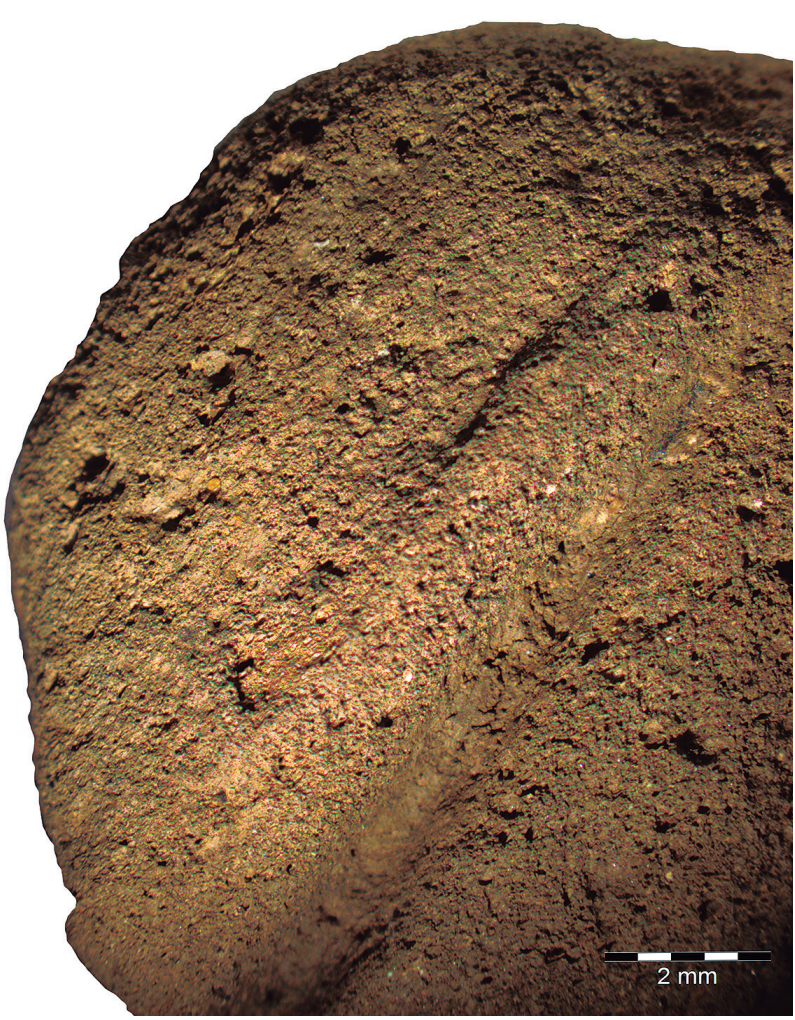

Fig.2. Desgaste en la parte izquierda de la cara A del pulidor $\mathrm{n}^{0} 1$ de Santa Catalina. / Wear on the left part of side A of Polisher No. 1 from Santa Catalina.

La cara B, a la derecha de la anterior, no conserva huellas de manipulación antrópica; tiene acusados huecos y cavidades naturales de perímetro redondeado.

Girando de nuevo a la izquierda situamos la tercera cara (C). Es de superficie irregular y en ella se aprecia un único surco ancho y alargado, algo menos profundo que los de la cara A. Nace del borde inferior y se desarrolla en diagonal hasta el borde lateral izquierdo, sin llegar a alcanzar el extremo superior. Tiene una longitud de 33,4 mm y una sección en $U$.

La base es plana, subcuadrangular, con bordes irregulares de forma sinuosa. En su parte central se observa un surco que la atraviesa en diagonal, de lado a lado. Tiene una longitud de 31,4 mm y es el más hondo de toda la pieza. Su sección es en $U$, con bordes rectos y paralelos (Figura 3).

En la parte izquierda del surco, se aprecia un hundimiento de bordes curvilíneos, perpendicular a la acanaladura central. Parece tratarse de una saltadura no intencionada, dado que no se observan en él huellas de regularización (Figura 3).

La observación microscópica ha permitido reconocer en las paredes y en el fondo de los surcos numerosas estrías rectilíneas de delineación longitudinal, paralelas a las aristas de las acanaladuras, que indican una acción de frotado de un objeto dentro de ellos con un movimiento bidireccional de va y viene.

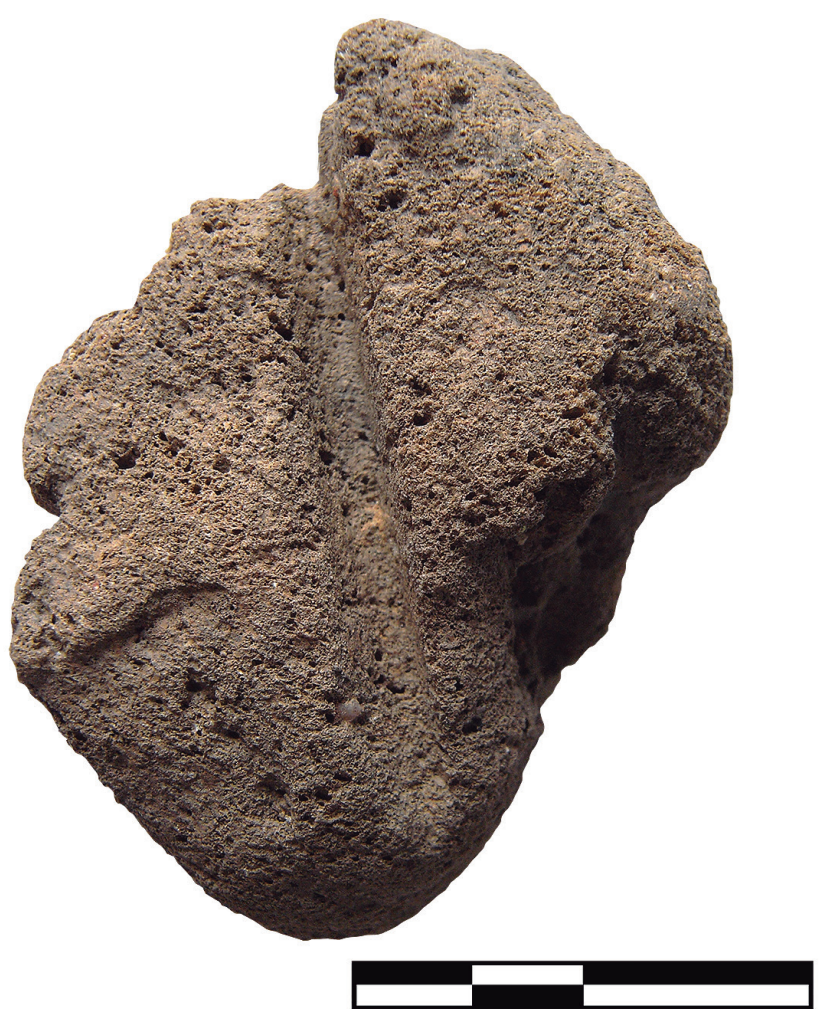

Fig.3. Base del pulidor $n^{\circ} 1$ de Santa Catalina. / Base of Polisher No. 1 from Santa Catalina.

En la parte inferior de las aristas de los surcos de la cara A se observan planos de desgaste, oblicuos a su dirección longitudinal, que se prolongan por la superficie de la cara, en los que hay señales de desgaste (Figura 4).

\section{Pulidor $n^{\circ} 2$ (SC.C8.154.721)}

Fragmento de forma globular y contorno ovalado. Sus dimensiones son: $38,4 \mathrm{~mm}$ de eje mayor, $28,4 \mathrm{~mm}$ de anchura máxima, $20 \mathrm{~mm}$ de espesor y el peso 7,51 gr. Estructura esponjosa con vesículas de mayor diámetro que las de la pieza anterior. Color grisáceo, con tonos azulados. En su estado actual tiene una saltadura superficial en una de sus caras, que debemos considerar de carácter postdeposicional (Figura 5, A). Junto a él se recuperó una pequeña esquirla de 0,35 gr de masa, de características petrológicas similares sin ningún tipo de marcas, que no ha podido ser integrada en esta pieza.

En uno de los lados del contorno se aplana formando una cara horizontal. En su zona central hay un surco rectilíneo de delineación vertical y poca profundidad, que la atraviesa de extremo a extremo. La hendidura tiene sección abierta, que podría asimilarse a una forma en $U$, con bordes rectos paralelos y fondo plano. Sus dimensiones son $27,9 \mathrm{~mm}$ de longitud y $4,8 \mathrm{~mm}$ de anchura (Figura 5, B). 


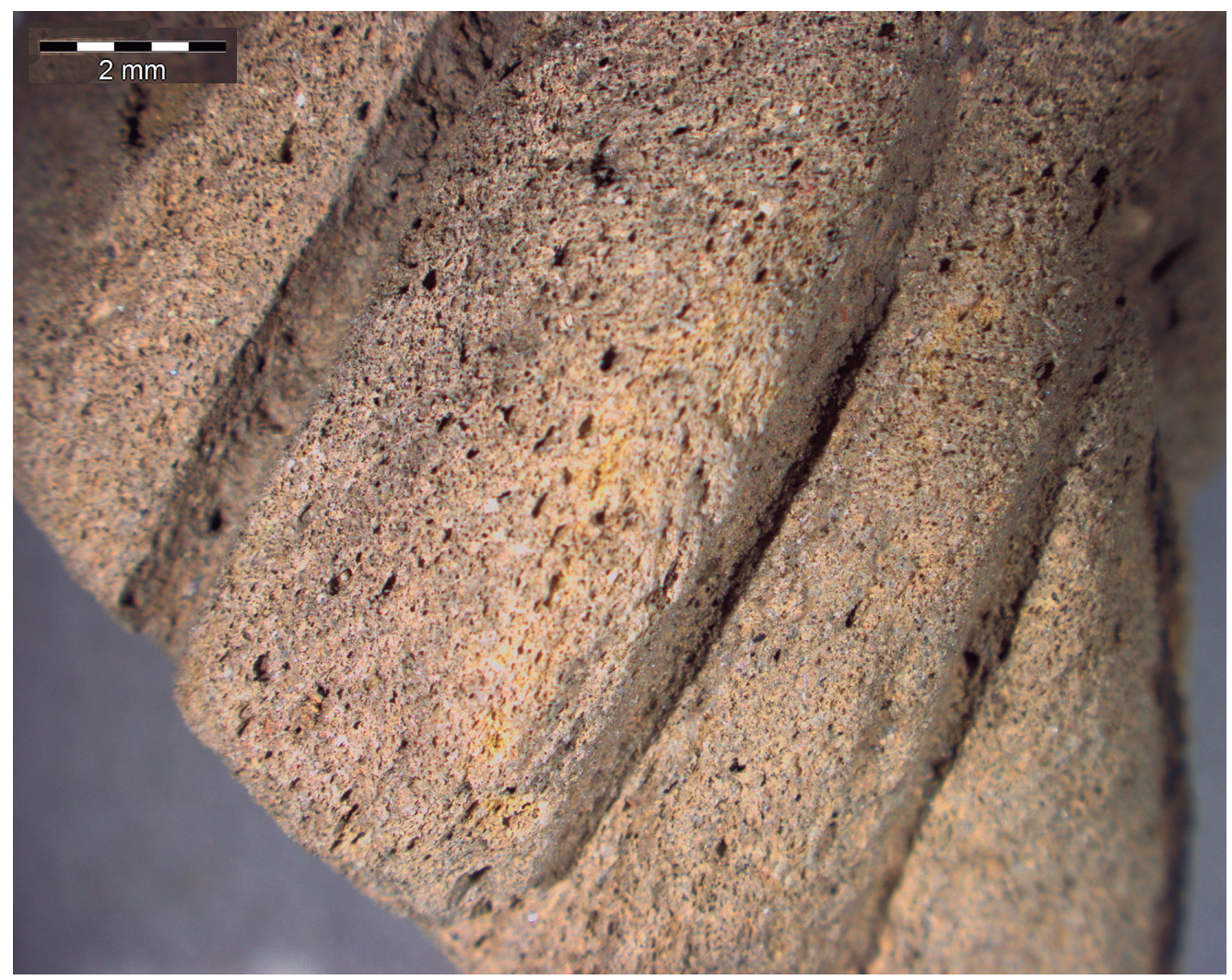

Fig.4. Desgastes en la parte inferior de las aristas de los surcos de la cara A del pulidor $n^{\circ} 1$ de Santa Catalina. Wear on the bottom of the edges of the grooves on side A of Polisher No. 1 from Santa Catalina.
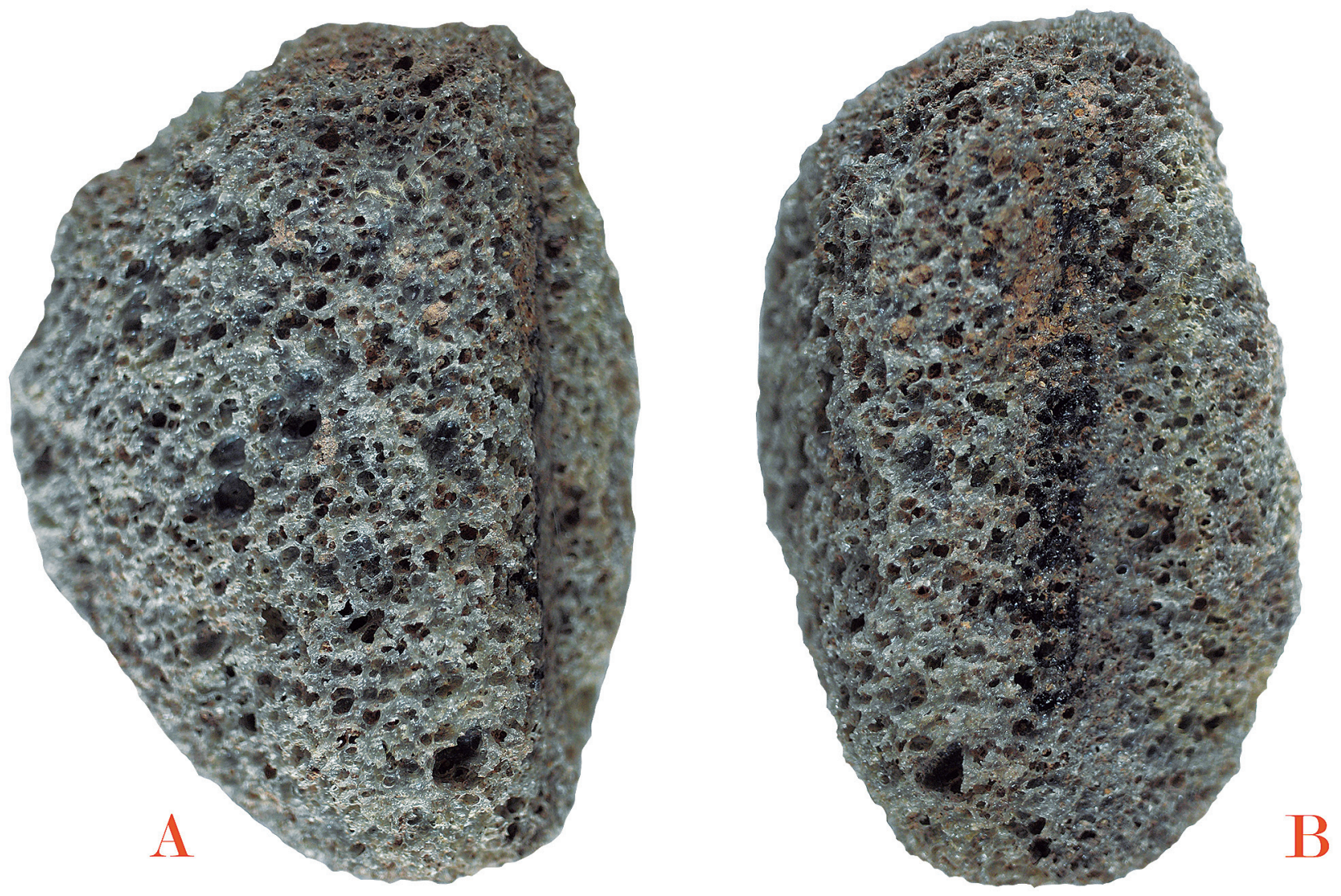

Fig.5. Pulidor $n^{\circ} 2$ de Santa Catalina. / Polisher No. 2 from Santa Catalina. 
El análisis a través de la lupa binocular ha permitido apreciar estrías de poca entidad en el interior del surco. Este hecho unido a la leve profundidad del mismo induce a suponer que fue usado en una acción esporádica de fricción, por lo que el desgaste producido es escaso.

\subsection{Cueva de Aitzbitarte IV}

Pulidor Aitz.3 Ø.2532? ${ }^{1}$.

Objeto recuperado durante la campaña de excavación de 1963 en cuya memoria se atribuye al nivel IV y se describe como "piedra pómez con orifico de suspensión y surcos de uso" (Barandiarán, J.M., 1964:19). En la síntesis estratigráfica y cultural del yacimiento realizada por I. Barandiarán, (1967:90) este nivel IV de 1963, lo asignó al nivel III de su secuencia estratigrafía publicada con una atribución cultural al Magdaleniense.

Es una pieza piramidal, con base de forma más o menos cuadrangular y extremo superior apuntado, con el ápice redondeado. El color es grisáceo, con tonos amarillentos y la estructura esponjosa; en ella se aprecian, de forma macroscópica, poros o vesículas de pe-
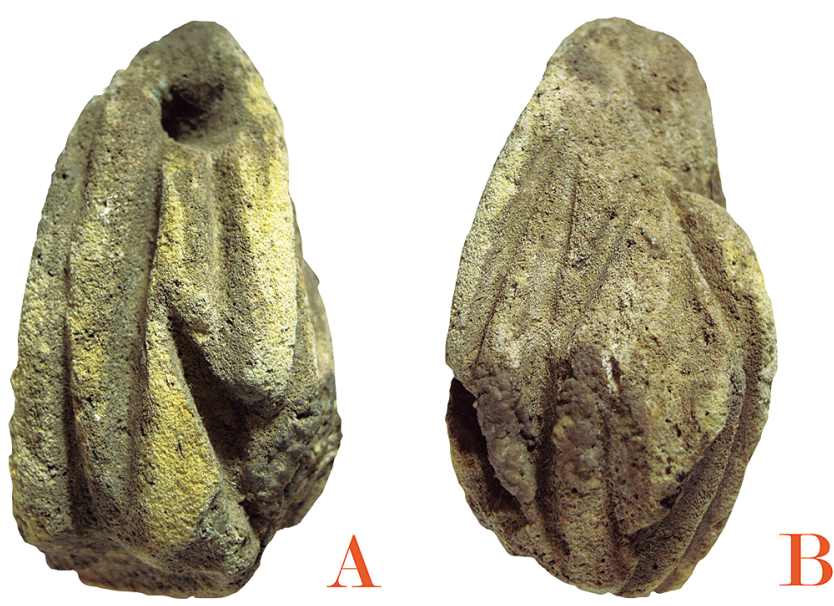

El estado de conservación es bueno. En pequeñas áreas de la superficie y en el interior de alguno de los surcos se observan adherencias calcáreas de poca entidad. En la parte inferior de la cara D hay una pequeña saltadura concoide, que parece el resultado de un golpe accidental, probablemente de origen postdeposicional.

En la parte distal, cerca del ápice, está perforado con un orificio que atraviesa desde la cara A hasta la D. La abertura en la cara A tiene un perímetro subcircular de 7,4 × 8,3 mm. En la zona derecha del borde de la arista se ha producido un desgaste que genera un plano inclinado, prolongándose por la superficie exterior de la cara. En su zona superior hay tres pequeñas saltaduras concoides, dos en el lado izquierdo y uno en el derecho (Figura 7.1). La parte inferior está afectada por el extremo de dos acanaladuras que interrumpen la delineación de su perímetro.

En el otro extremo de la perforación, la abertura es más regular y de perímetro circular $(6,4 \mathrm{~mm} \varnothing)$. El contorno de la arista, al igual que en la cara A, presenta alteraciones. Se aprecian pequeñas saltaduras escamosas concoides en la parte superior, dos continuas en el

Fig.6. Pulidor de Aitzbitarte IV. / Polisher from Aitzbitarte IV.

queño tamaño y algunas cavidades algo mayores. El aspecto y la estructura se asemejan a los del pulidor $n^{\circ}$ 1 de Santa Catalina. Las dimensiones son: 58,4 mm de altura y $33 \mathrm{~mm}$ de anchura máxima; el peso es de 17,67 gr. Hemos diferenciado cuatro caras, que identificaremos con las letras A a D; tres de ellas son de superficie convexa poco marcada y la cuarta más aplanada, ligeramente hundida (Figura 6).
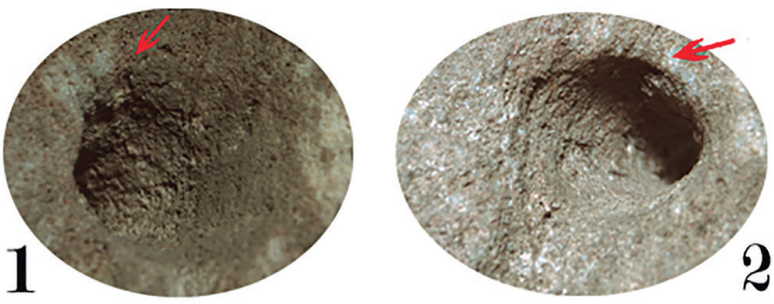

Fig.7. Pulidor de Aitzbitarte IV: 1. Abertura en la cara A. 2. Abertura en la cara D. / Polisher from Aitzbitarte IV: 1. Opening on side A. 2. Opening on side D.

\footnotetext{
${ }^{1}$ Sigla con que se registra en la etiqueta de Gordailua.
} 
lado izquierdo y una aislada en el derecho, de las que se ha desprendido una porción mínima de materia. La parte inferior, al igual que en la otra abertura, está afectada por el extremo de una acanaladura (Figura 7.2).

Las alteraciones producidas por los surcos en ambas aberturas, indican que su uso fue posterior a la perforación.

El interior de la perforación tiene un perfil bicónico. En sus paredes se observan estrías circulares concéntricas, que marcan el sentido de la rotación realizada en su conformación y estrías orientadas hacia el interior del cono, que se superponen a las anteriores.

Como en el caso del pulidor $n^{\circ} 1$ de Santa Catalina, la descripción de las caras la haremos girando la pieza hacia la izquierda en el orden que hemos establecido en la figura 6.

Sobre la superficie de la primera (A) se marcan con nitidez cuatro surcos de diferente orientación, tamaño y profundidad. Los dos de la izquierda, los de mayor longitud, son paralelos entre sí, tienen trayectoria longitudinal y se extienden de base a vértice, si bien solo uno de ellos lo hace hasta la arista inferior del orificio. El tercero, también longitudinal y paralelo a los anteriores, ocupa la zona derecha; tiene un desarrollo más corto que se inicia en la zona medial del fragmento, donde converge con la arista del cuarto, y alcanza el borde de la perforación. El cuarto tiene una orientación oblicua y se desarrolla desde la intersección del borde inferior con el lateral derecho hasta la zona central de la cara. Es el más profundo de todos ellos y en su arista derecha presenta una saltadura triangular con pérdida de materia, que interrumpe su regularidad lineal. Los dos primeros presentan una sección en $U$, mientras que los otros dos la tienen en $\mathrm{V}$. En todos se observan nítidas estrías longitudinales en el interior del surco (Figura 6).

La cara B, salvo la zona apuntada del extremo distal, está completamente surcada por acanaladuras de diferente longitud y orientación, que no se cruzan entre sí. Son ocho hendiduras en su mayoría de delineación longitudinal. Los surcos no tienen la misma profundidad, ni esta es homogénea dentro de cada uno, puesto que en alguno de ellos hay zonas más hundidas que otras. Seis tienen sección en U y dos en V (Figura 6).

La cara $\mathrm{C}$ está atravesada por tres ranuras rectilíneas paralelas, de tamaño bastante similar, que la abarcan en su totalidad. La profundidad varía notablemente entre las dos situadas a los lados, más anchas y profundas, especialmente la de la derecha, y la situada entre ellas, menos profunda y más estrecha. La sección de las primeras es en $U$ y la de la tercera en $V$, aunque en la parte distal se ancha levemente, tendiendo a una forma más abierta. En las paredes de todas ellas se aprecian marcadas estrías longitudinales.

Cerca del ápice, se aprecia una depresión de perímetro circular (5,6 mm Ø), forma interior cóncava y fondo plano. En la pared son visibles de forma macros- cópica estrías de delineación circular y, en las proximidades de la base, un escalón formado por una mínima rebaba o resalte. El contorno de la arista del borde está alterado, en su parte superior, por dos saltaduras concoides, irregulares, una de ellas más profunda que la otra, que, por sus dimensiones, parecen ser el negativo producido por el desprendimiento de sendas lascas durante la acción de horadar la roca. (Figura 8).

En las aristas de dos de los surcos y en una zona de la superficie no alterada por la acción antrópica, destacan unas manchas anaranjadas que parecen ser adherencias de una materia colorante, quizás ocre, que ha teñido la superficie incrustándose en algunas de sus oquedades. No afectan ni a las paredes ni a la base de las acanaladuras (Figura 6 y 10).

Sobre la última (D) se han generado dos ranuras longitudinales paralelas entre sí de diferente entidad. La central, de sección en $V$ en la parte inferior, alcanza la arista del borde de la perforación donde la sección se ensancha; su desarrollo es corto y se extiende desde el borde de la perforación hasta la zona media de la cara. La de la derecha, de sección en V, con zonas algo más anchas que se acercan a una sección en forma de $U$, recorre la totalidad de la cara de extremo a extremo (Figura 6).

La base está atravesada por dos hendiduras paralelas, profundas y rectilíneas. La más honda tiene sección en $U$ y se conserva intacta. El grado de deterioro de la otra dificulta establecer su sección original. Uno de los surcos de la cara A desborda el límite inferior de la base y afecta parcialmente a su superficie, alterando las marcas que se habían producido en ella. (Figura 9).

\subsection{Interpretación funcional}

Como hemos indicado en la descripción de los pulidores hemos visto en ellos diferentes tipos de huellas, que consideramos ocasionadas por su uso como herramienta. En el fondo y en las paredes de los surcos hay abundantes estrías longitudinales y un alisado y regularización del microrrelieve original de la roca. Sobre algunos tramos de las aristas se han generado planos oblicuos en los que también hay estrías, consecuencia de un rozamiento que las ha modificado, rebajando tanto la pared del surco como su borde. Todas estas alteraciones parecen responder a los efectos de un trabajo de frotamiento con presión de materiales duros sobre la superficie mediante un movimiento longitudinal y, quizás, también rotatorio, con el fin de obtener el afinado y pulido de los mismos. Las desviaciones que se observan en los laterales de algunas de las acanaladuras las interpretamos como líneas de fuga o movimientos poco o mal controlados acaecidos durante la realización de esa labor.

En la cara A del pulidor $n^{\circ} 1$ de Santa Catalina se distingue una superficie alterada por desgaste con unas leves, pero nítidas, estrías longitudinales, en la 


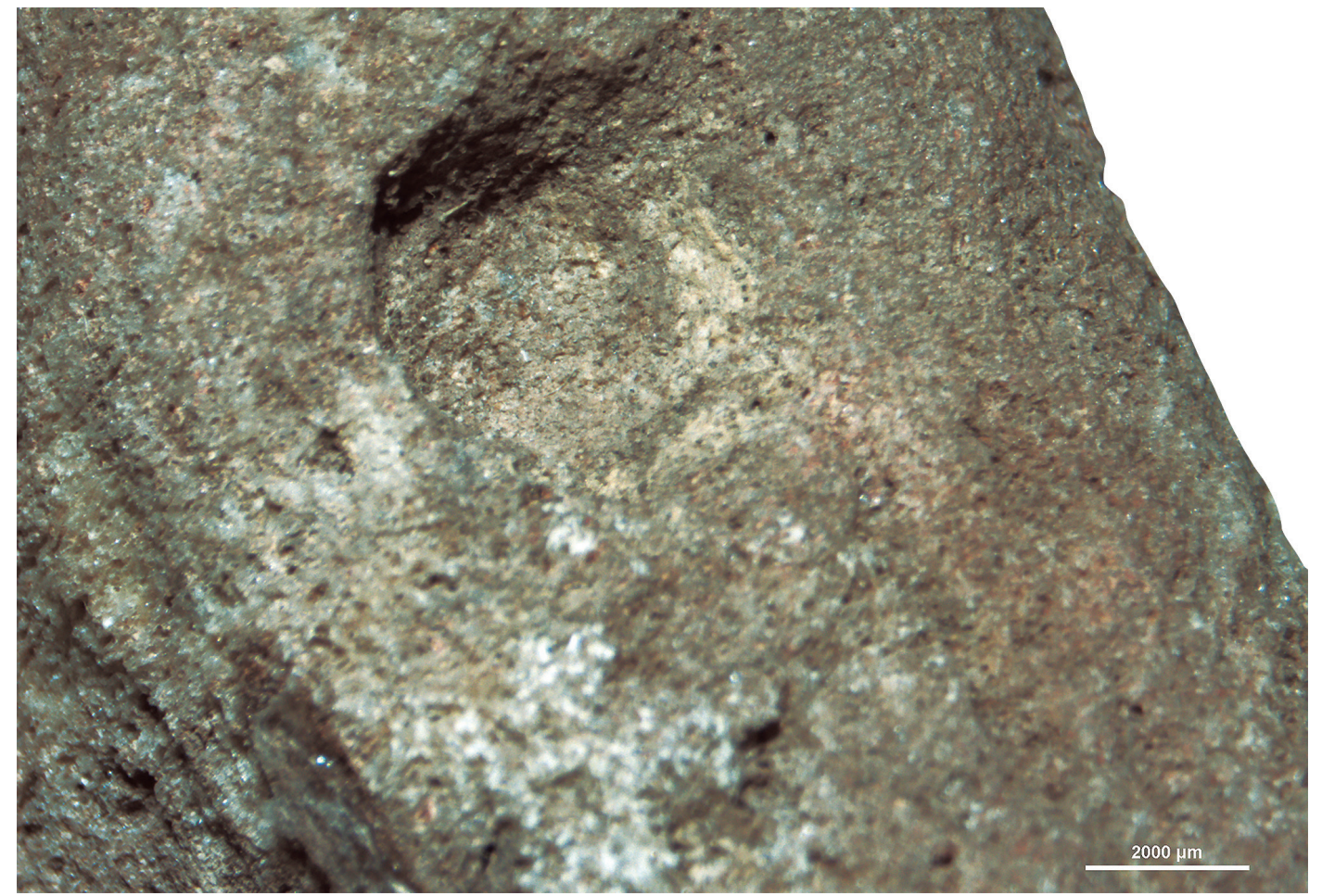

Fig.8. Depresión circular en la cara C del pulidor de Aitzbitarte IV. / Circular depression on side $\mathrm{C}$ of the polisher from Aitzbitarte IV.

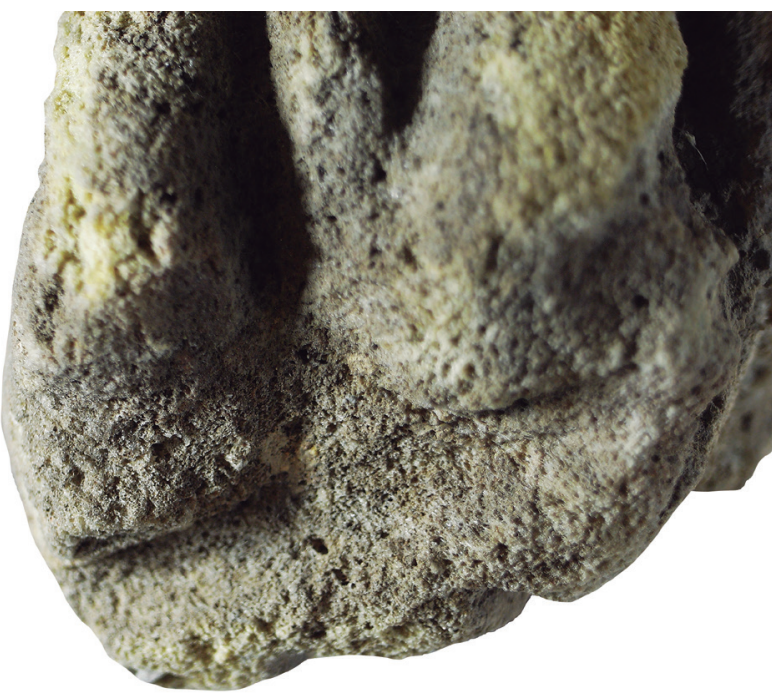

Fig.9. Base del pulidor de Aitzbitarte IV. / Base of the polisher from Aitzbitarte IV.

que no se ha llegado a formar una hendidura. La actividad que se realizó en esa zona debió de ser similar a la que acabamos de describir, aunque de menor intensidad, por lo que las huellas resultantes son más someras (Figura 2).

El pulidor de Aiztbitarte IV presenta claras semejanzas con el anterior. Todas sus caras han sido usadas reiteradamente hasta generar en ellas numerosos surcos, algunos de notable hondura. El aspecto de las huellas que se observan en las acanaladuras es similar al del pulidor anterior y responde a una tarea análoga a la descrita.

Los pulidores con ranuras, indistintamente de la materia prima que fuera seleccionada, se han relacionado con algunas fases del trabajo de conformación de determinados equipamientos en materias óseas animales (Beaune, 2000 y 2012). Esa función es el origen de los desgastes que se producen en las zonas activas de las piezas hasta el punto de causar una importante pérdida de materia en las mismas. Por otra parte, la anchura de los surcos parece indicar que debemos relacionarlos con el acabado o afinado de piezas estrechas y alargadas. El hallazgo de este tipo de útil en el nivel III de Santa Catalina está en consonancia con la abundante industria ósea recuperada en él, entre la que son numerosas las agujas y los apuntados (Berganza et al., 2012). No cabría descartar de forma absoluta la hipótesis de su empleo sobre otras materias no tan duras, aunque sí lo suficientemente consistentes como para originar ese tipo de huellas. En ese mismo nivel se han recuperado dos cuentas de madera completamente redondeadas en su contorno, cuyo acabado ha requerido una tarea de regularización, que podría haberse hecho con un material abrasivo como la pumita (Berganza y Ruiz Idarraga, 2018). Lo mismo podría decirse del pulidor del nivel III de Aitzbitarte IV, en el que se encontraron apuntados, algún punzón y una aguja (Mujika, 1983).

Los programas experimentales que han reconstruido el proceso de fabricación y mantenimiento de agujas paleolíticas han verificado que, en algunas de 
las fases de elaboración de esos útiles, se emplearon cantos de arenisca sobre los que se frotaron los objetos óseos con la finalidad de conseguir su desgaste o afinado. Tanto en la forma como en las huellas que se han producido en los objetos experimentales, se aprecian notables similitudes con las que se observan en las piezas arqueológicas (Corchón Rodríguez y Garrido Pimentel, 2007; Laborda, 2013).

La presencia de desgastes superficiales fuera de las acanaladuras en el pulidor $n^{\circ} 1$ de Santa Catalina, puede que se deba al inicio de un surco no continuado y, por lo tanto, no ahondado por la repetición del gesto técnico realizado.

La presencia de adherencias de materia colorante, posiblemente ocre, en la cara $\mathrm{C}$ del pulidor de Aitzbitarte IV, que afectan a la parte superior de las aristas de dos de los surcos y a una zona de la pieza sin marcas de desgaste es un hecho destacable. Es difícil determinar si se embebieron en las vesículas de la piedra pómez porque se empleó un aditivo mineral en los procesos de transformación en los que se empleó o si se debe a un teñido accidental que se produjo en el yacimiento por proximidad a un residuo de ese colorante (Figura 10).

La perforación que tiene este pulidor, así como lo que puede interpretarse como un intento de perfo- ración inacabado en la cara $\mathrm{C}$, es un rasgo peculiar del mismo. En el primero se aprecian tanto las marcas ocasionadas por la rotación de un útil lítico, muy posiblemente de sílex, como las que se les superponen ocasionadas por su presumible función. Las saltaduras de la parte superior de los extremos del orificio, así como las estrías que se orientan desde el interior hacia el exterior de sus paredes muestran el efecto erosivo ocasionado por un constante movimiento de suave fricción, que debió producirse al estar suspendido por medio de una ligadura. Al tratarse de un instrumento de pequeño tamaño, la sujeción a otro elemento debió de ser un recurso para evitar su extravío o, quizás también, facilitar su óptimo manejo como herramienta.

En el orificio que quedó inconcluso son claramente visibles las marcas de la rotación realizada para horadar la pumita.

El pulidor $n^{\circ} 1$ de Santa Catalina y el de Aitzbitarte IV son útiles poliranurados con surcos profundos que indican un uso intenso. Las huellas del pulidor $n^{\circ} 2$ de Santa Catalina, por el contrario, evidencian un uso más limitado. Todos conservan algunas zonas de la potencial superficie abrasiva no afectadas por desgaste, lo que hace suponer que fueron abandonados en una fase previa a su total agotamiento.

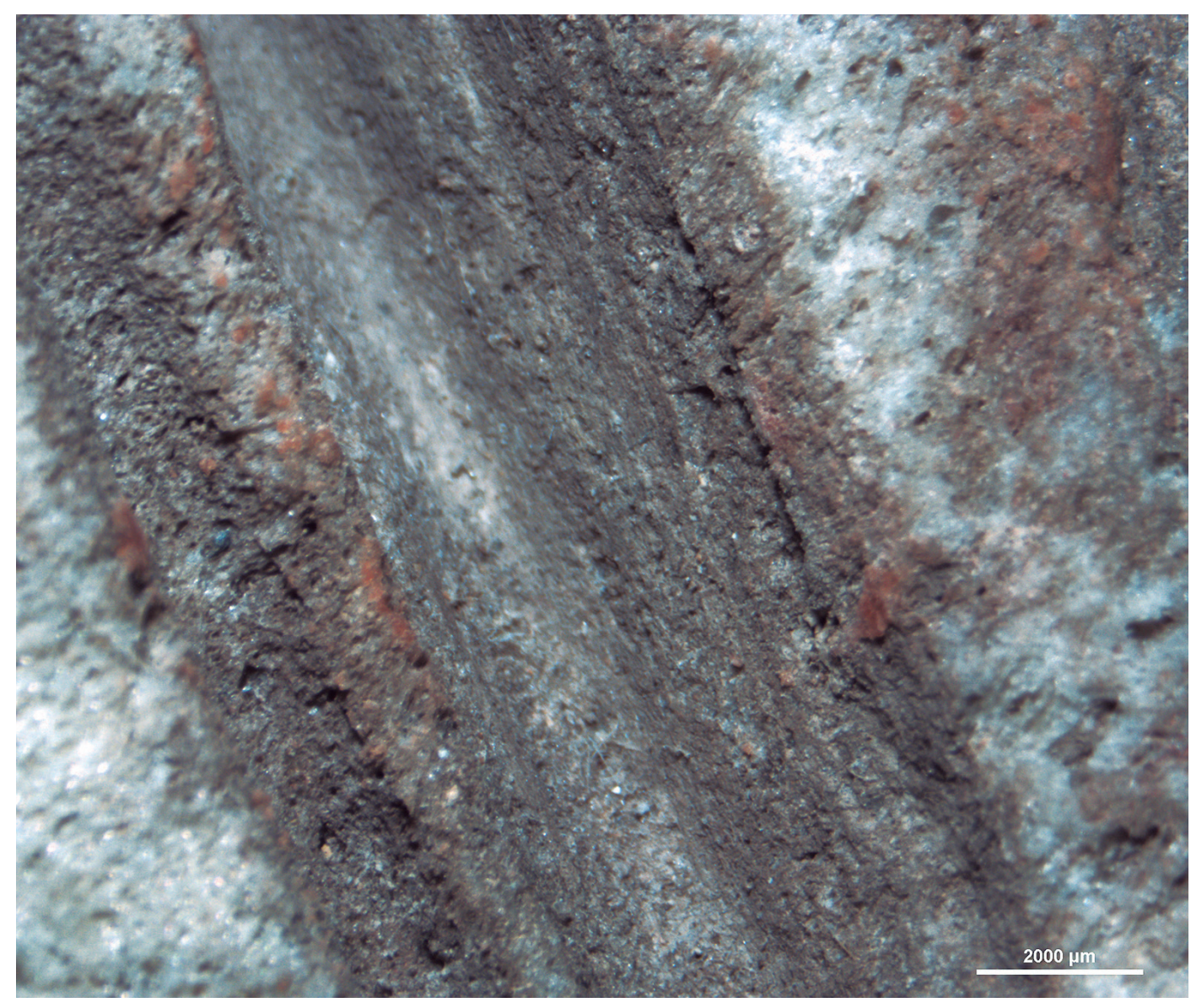

Fig.10. Rastros de colorante sobre la cara $\mathrm{C}$ del pulidor de Aitzbitarte IV. I Traces of dye on side $\mathrm{C}$ of the polisher from Aitzbitarte IV. 


\section{PROCEDENCIA DE LA MATERIA PRIMA}

El estudio de los útiles de piedra pómez suscita, en todos los yacimientos arqueológicos en los que han sido hallados, la cuestión de determinar, con la mayor precisión posible, la procedencia de esa roca. Para los asentamientos emplazados en la vertiente atlántica del continente europeo, tanto con secuencias del Pleistoceno Superior como holocénicas, se proponen dos potenciales zonas geográficas de origen asociadas a fenómenos de erupción volcánica: Islandia y el Caribe (Giot, 1972; Binns, 1972; Donnart, 2007; Dachary et al., 2012; Lefort, 2014). El análisis geoquímico de cada objeto permite precisar la composición del mismo y, a partir de esa información, inferir, con mayor probabilidad, su procedencia concreta (Binns, 1972; Dachary, et al., 2012). En las piezas que presentamos no disponemos de esa información, por lo que no podemos trascender del planteamiento hipotético general sobre su origen en los focos volcánicos nórdico o subtropical.

Teniendo en cuenta la flotabilidad de estos materiales, debemos considerar que su llegada a las costas del Golfo de Bizkaia, se produjo por el transporte de alguna corriente marina procedente del Ártico o del Caribe (Corriente del Golfo).

En el caso de Santa Catalina lo más plausible es considerar que las pumitas fueran recogidas en las playas próximas. En este asentamiento hay abundantes datos de que sus ocupantes vivieron orientados hacia el mar y explotaron los recursos que este medio les ofrecía. Cabe mencionar como referencias significativas la abundante pesca de variadas especies marinas (Roselló et al., 2014 y 2015), la inusual recogida de un número considerable de restos de cetáceos y la captura de focas (Castaños, 2014), el también singular hallazgo de una valva de Chlamys islandica decorada con motivos geométricos (Berganza et al., 2012), el testimonio que aporta la recogida de algas (Vásquez y Rosales, 2014), etc.

\section{LOS PULIDORES DE PIEDRA PÓMEZ EN LA CORNISA CANTÁBRICA Y EL PIRINEO OCCI- DENTAL}

Los pulidores con ranuras sobre piedra pómez que se estudian en este artículo son, hasta el momento, las tres únicas referencias de hallazgos de este tipo en la Cornisa Cantábrica.

En la vertiente norte del Pirineo occidental han sido localizados en 4 yacimientos próximos a la costa atlántica atribuidos a ocupaciones del Paleolítico superior. Son los sitios de Arancou (Bonnisset y Chauvière, 1999), Duruthy (Dachary et al., 2012), Isturitz (Passemard, 1944; Beaune y Buisson, 1996; Beaune, 2003) y Gourdan (Piette, 1873) (Figura 11).

En todos estos lugares son elementos excepcionales dentro de sus amplios registros arqueológicos. En cuatro hay un único ejemplar y en los otros dos, Isturitz y Santa Catalina, se han recuperado un par de ellos. La forma, tamaño y volumen de los objetos es bastante similar, con alguna variedad en la dimensión de sus poros, lo que, muy posiblemente, se explique no tanto por una selección antrópica cuanto por las propias características de la pumita a su alcance.

Los asentamientos están situados en un territorio relativamente reducido. Como se aprecia en la figura 11, cuatro se localizan en el suroeste de Francia, tres en las proximidades del cauce medio-bajo del Adour y el cuatro, el más oriental, en el alto Garona. Los dos restantes, en el norte de España, uno en el curso bajo del Urumea y el otro, el más occidental, en un acantilado calizo sobre el Mar Cantábrico, junto a la desembocadura actual del río Lea.

Los yacimientos del sur de los Pirineos son los más cercanos al litoral mientras que en los del norte esa distancia es algo mayor, sobre todo en el caso de la cueva de Gourdan, que puede considerarse un lugar de habitación interior. La proximidad o lejanía con respecto a la línea de costa en el final del Pleistoceno Superior debe evaluarse en función del impacto causado por la regresión marina de la última glaciación y de las características particulares de la plataforma continental emergida en cada uno de los tramos de la costa del Golfo de Bizkaia. Ese espacio, hoy sumergido bajo las aguas, fue de mucha mayor amplitud en la costa atlántica que en la cantábrica. En consecuencia, las cuevas de Aitzbitarte IV y de Santa Catalina fueron durante el Tardiglaciar las más cercanas al mar y a los recursos que este pudiera proporcionar, entre los que debemos incluir los fragmentos de piedra pómez. Para el caso de ese último, los cálculos realizados sitúan la línea de costa a poco más de $5 \mathrm{~km}$ mar adentro con respecto al litoral actual.

La cercanía a la costa de la mayor parte de ellos debió de ser un factor de accesibilidad que posibilitó la captación de un recurso exótico en esta región. El emplazamiento más favorable para la práctica de esa recolección fue, sin duda, el de Santa Catalina, Iocalizado en un promontorio frente al mar desde el que se tendría un acceso fácil a su orilla. En el resto de los casos, la distancia a recorrer hasta la costa fue algo mayor, aunque sólo de algunas decenas de km. En consecuencia, podemos afirmar que las comunidades prehistóricas que usaron este recurso, se sirvieron de una materia prima de carácter "local" por el entorno en el que era captada. El ejemplar hallado en la parte central del Pirineo, en sentido contrario, atestigua un alejamiento entre las áreas de captación y el lugar de uso y abandono, lo que permite inferir una circulación oeste-este a lo largo del territorio pirenaico (Dachary et al., 2012). Esta movilidad también ha sido documentada para las cabezas de proyectil de gran tamaño elaboradas sobre hueso de cetáceo cuya procedencia es indudablemente atlántica (Petillon, 2013), el transporte de materias primas líticas (Tarriño et al., 2015, 2016) o 
en las similitudes técnicas y estilísticas observadas en el arte mueble (Rivero, 2010; Fano y Rivero, 2012). La falta de pulidores en la mitad oriental de los Pirineos apunta hacia la escasa probabilidad de unas relaciones a larga distancia e incide en descartar la procedencia mediterránea de las pumitas.

Cabe plantearse la cuestión de determinar si la elección de esta materia fue el resultado de una práctica oportunista o si, por el contrario, hubo una búsqueda selectiva de la misma. El pequeño número de pulidores en piedra pómez y su reducido porcentaje en relación con instrumentos similares sobre otros tipos de roca podría indicar o bien la escasez de esa materia o bien la menor idoneidad para la función a la que eran destinados. El aprovechamiento intensivo que se observa en estos objetos parece manifestar que era un material óptimo para esa función. La excepcional perforación del ejemplar de Aitzbitarte IV podría considerarse un indicador de la estima de esa herramienta.

Alguno de los ejemplares referidos tienen una contextualización cronoestratigráfica precisa caso de los de la cueva de Santa Catalina, hallados en un nivel datado en una horquilla temporal, entre el 12900 y el 15000 Cal BP, que se corresponde con la de otros asentamientos cantábricos atribuidos a las fases finales del Magdaleniense. Una cronología algo más antigua, entre el 15000 y el 16400 Cal BP, ha sido determinada para el nivel 1/F1 de la cueva de Isturitz del que procede el pulidor magdaleniense de ese yacimiento, fecha que se sitúa en los comienzos del Magdaleniense superior (Petillon et al., 2009).

El otro pulidor de este último yacimiento ha sido asignado a un nivel auriñaciense. Sin embargo, en sucesivas revisiones del utillaje en piedra no tallada S.A. de Beaune (1996, 2000 y 2017) ha cuestionado esa atribución, considerando más razonable vincularlo a una cronología magdaleniense.

El de Aitzbitarte IV, si bien carece de datación, se ha recuperado en un nivel que algunos autores, atendiendo a los conjuntos industriales lítico y óseo, atribuyen al Magdaleniense (Barandiaran, 1967).

El resto aunque no han sido recuperados en secuencias estratigráficas precisas, han sido asignados a conjuntos tecnoindustriales Magdalenienses (Dachary et al. 2012).

\section{CONCLUSIÓN}

El estudio de los útiles líticos no tallados aporta información sobre tareas técnicas y domésticas desarrolladas en los asentamientos paleolíticos. Está constatada la utilización de rocas diversas en las cadenas operativas para la conformación de útiles en piedra y en materias duras animales, para el trabajo de la piel o el machacado y molienda de vegetales. En las excavaciones de la cueva de Santa Catalina se ha recuperado una notable colección de objetos de estas características en la que están presentes varios de los tipos que

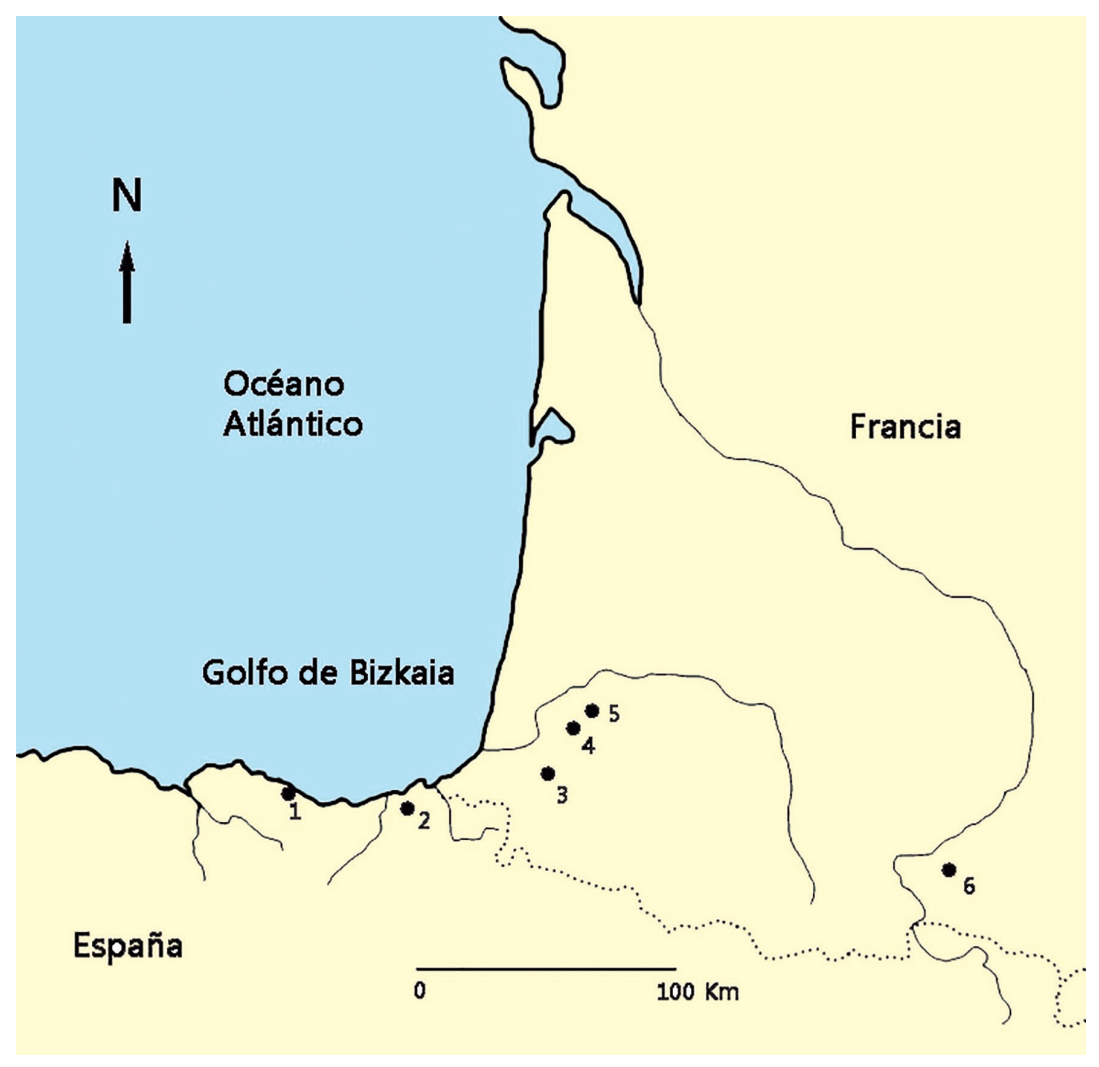

Fig.11. Mapa de dispersión de los pulidores en piedra pómez (1 Santa Catalina; 2 Aitzbitarte IV; 3 Isturitz; 4. Arancou; 5 Duruthy; 6 Gourdan). / Map of the dispersion of pumice stone polishers (1 Santa Catalina; 2 Aitzbitarte IV; 3 Isturitz; 4. Arancou; 5 Duruthy; 6 Gourdan). 
han sido establecidos para esa categoría de instrumentos (Arribas y Berganza, 2018).

En dos ejemplares de esa colección se aprecian improntas definitorias de los denominados pulidores con ranuras, con la peculiaridad de que se trata de fragmentos de piedra pómez (pumita). Idénticas huellas muestra una pieza, también de piedra pómez, procedente del yacimiento de Aitzbitarte IV.

El empleo de esa materia prima, que no forma parte de la composición geológica del ámbito geográfico en el que se sitúan los asentamientos, pero que por su flotabilidad ha podido ser arrastrada por las corrientes marinas hasta las costas del Golfo de Bizkaia, sugiere una estrategia de captación de un recurso accesible en un medio litoral.

El análisis de las huellas nos ha permitido determinar que las pumitas se emplearon para frotar una materia dura con el fin de fabricar o reavivar un útil. La fricción entre ambos materiales provocó una abrasión en la roca, que fue produciendo un desgaste con pérdida de materia hasta generar los surcos o acanaladuras que caracterizan a estos utensilios. Los programas experimentales relacionan los pulidores con ranuras con la fabricación de agujas u otros apuntados en hueso, asta o, quizás, madera. Esa función debió de ser la desarrollada por los de Santa Catalina y Aitzbitarte IV. Su presencia en niveles en los que se hallaron restos del trabajo de fabricación de útiles en hueso y asta parece indicar que las labores en los que intervinieron se realizaron en el propio lugar.

En el caso de Santa Catalina, esta evidencia se suma a la proporcionada por las huellas de uso registradas en la industria lítica en sílex, que muestran una importante actividad relacionada con el trabajo de materias óseas. La asociación de las estrategias de captación de recursos especializados, las actividades domésticas de transformación de materias duras animales o de piel seca, entre otras labores que requieren una importante dedicación de tiempo y esfuerzo, inciden en reforzar la hipótesis ya manifestada de que las estadías que se produjeron en la cueva durante el Magdaleniense fueron prolongadas. (Ibáñez Estévez, J. J. et al., 1993; Ibáñez Estévez, J. J. y González Urquijo, J. E., 1996).

La búsqueda bibliográfica de otros pulidores con ranuras en piedra pómez, nos ha permitido constatar que el repertorio de los mismos, con los datos de que se disponen en la actualidad, es reducido. Por otra parte, el territorio en el que se localizan se circunscribe al suroeste de Francia, junto a la Cordillera pirenaica, y a la zona este de la Cornisa Cantábrica, por lo que puede considerarse un área geográfica relativamente limitada. No se refieren hallazgos más allá de los Pirineos centrales, ni en el resto del norte de la Península Ibérica. Los sitios en los que se han producido los hallazgos se encuentran relativamente próximos entre sí y no distanciados en exceso de la línea de costa del Golfo de Bizkaia a finales del Pleistoceno.
La recogida de la pumita, así como la captación de otros recursos que hemos ido señalando, vincula a las comunidades de finales del Pleistoceno Superior que ocuparon los valles costeros y las llanuras litorales de esa región con el conocimiento y el aprovechamiento de la rica variedad de elementos que proporciona un ecosistema marino.

\section{BIBLIOGRAFÍA}

Arribas, J. L., Berganza, E., 2018. La dieta vegetal en la cueva de Santa Catalina (Lekeitio, Bizkaia). Cantos con huellas de procesado de vegetales. Kobie Anejos 18, 245-254.

Barandiarán Maeztu, I., 1967. El Paleomesolítico del Pirineo Occidental. Bases para una sistematización tipológica del instrumental óseo paleolítico. Monografías Arqueológicas vol. 3. Zaragoza

Barandiarán, J. M., 1964. Excavaciones en la caverna de Aitzbitarte IV (campaña 1963). Munibe 16(1-2), 12-23.

Beaune, S. A. de, 2000. Pour une archéologie du geste. Broyer, moudre, piler, des premiers chasseurs aux prémieres agriculteurs. CNRS, Paris.

Beaune, S. A. de, 2014. Appréhender les outils simples en contexte préhistorique. Artefact 2, 137-148.

Beaune, S. A. de, 2017. Le matériel lithique non taillé de la grotte d'Isturitz issu des fouilles Passemard et Saint-Périer. En: Normand, Ch., Cattellain, P. (Eds.), La grotte d'Isturitz. Fouilles anciennes et récentes. Actes de la table ronde du cinquantenaire du classement comme Monument Historique des grottes d'Isturitz et d'Oxocelhaya, 25-34. Cedarc, Treignes. Artefacts 13.

Beaune S. A. de, Buisson D., 1996. Différenciation spatiochronologique de l'utilisation des galets au cours du Paléolithique supérieur pyrénéen: les cas d'Isturitz (Pyrénées-Atlantiques) et de La Vache (Ariège). In: Delporte, H., Clottes, J. (ed.), Pyrénées préhistoriques, arts et sociétés. Actes du 118e Congrès national des Sociétés historiques et scientifiques, 25-29 oct. 1993, Pau, 129-142. Éd. du Comité des Travaux scientifiques et historiques, Paris.

Berganza, E., Arribas, J. L. (Coords.), 2014. La cueva de Santa Catalina (Lekeitio, Bizkaia): La intervención arqueológica. Restos vegetales, animales y humanos. Kobie, serie BAI 4. Diputación Foral de Bizkaia- Bizkaiko Foru Aldundia, Bilbao.

Berganza, E., Ruiz Idarraga, R., 2018. Cuentas de madera magdalenienses de la cueva de Santa Catalina (Lekeitio, Bizkaia, España). Munibe Antropologia-Arkeologia 69, 63-73.

Berganza, E., Arribas, J. L., Ruiz Idarraga, R., 2012. Estudio tecnológico de los moluscos marinos perforados de los yacimientos de Lumentxa y Santa Catalina (Lekeitio, Bizkaia). Munibe Antropologia-Arkeologia 63, 93-104.

Berganza, E., Arribas, J. L., Castaño, P., Elorza. M., González Urquijo, J. E., Ibáñez, J. J., Iriarte, M.J., Morales, A., Pemán, E., Roselló, E., Uriz, A., Uzquiano, P., Vásquez, V., Zapata, L., 2012. La transición Tardiglaciar en la costa oriental de Bizkaia: el yacimiento de Santa Catalina. Resultados preliminares. En: Arias Cabal, P., Corchón Rodríguez, M. S., Menéndez Fernández, M., Rodríguez Asensio, J. A. (coords.), El Paleolítico Superior Cantábrico. Actas de la Primera Mesa Redonda, San Román de Candamo (Asturias), 26-28 de abril de 2007, 171182. Universidad de Cantabria, Santander. 
Binns, R. E., 1972. Composition and Derivation of Pumice on Postglacial Strandlines in Northern Europe and the Western Arctic. GSA Bulletin 83(8), 2303-2324.

Bonnisset, D., Chauvière, F.-X., 1999. L'industrie sur matières dures animale. In: Chauchat, C. (Dir.). L'habitat magdalénien de la grotte du Bourrouilla à Arancou (Pyrénées-Atlantiques). Gallia Préhistoire 41, 36-53.

Castaños, P., 2014. Estudio de los macromamíferos del yacimiento de Santa Catalina". En: Berganza, E., Arribas, J. L. (coords.), La cueva de Santa Catalina (Lekeitio, Bizkaia): La intervención arqueológica. Restos vegetales, animales y humanos. Kobie, serie BAl 4, 331-360. Diputación Foral de Bizkaia- Bizkaiko Foru Aldundia, Bilbao.

Corchón Rodríguez, M. S., Garrido Pimentel, D., 2007. Labores de mantenimiento y uso identificadas en las agujas de la cueva de las Caldas (Asturias, España). Zephyrus 60, 79-97.

Dachary, M., Deniel, C., Plassard, F., Boivin, P., Devidal, J.-L., 2012. Analyse textural et géochimique d'un polissoir à rainures du gisement magdalénien de Duruthy (Sorde, Landes, France). PALEO 23, 1-12.

Donnart, K., 2007. Le Macro-outillage. In: Yvan Pailler, D., Giovamacci, S., Ihuel, E., Tresset, A. (dir.), Beg Ar Loued: un habitat en pierres seches de la fin du Neololithique / Age du Bronze Ancien (volume texte)

Fano Martínez, M. Á., Rivero Vilá, O., 2012. El territorio y la movilidad de los cazadores del final del Paleolítico: algunas reflexiones metodológicas. En: Arias Cabal, P., Corchón Rodríguez, M. S., Menéndez Fernández, M., Rodríguez Asensio, J. A. (coords.), El Paleolítico Superior Cantábrico. Actas de la Primera Mesa Redonda, San Román de Candamo (Asturias), 26-28 de abril de 2007, 207-216. Universidad de Cantabria, Santander.

Giot, P.-R., 1972. La Pierre ponce des sites préhistoriques de Bretagne. BSPF 69, 159-160.

Ibáñez Estévez, J. J., González Urquijo, J. E., 1996. From Tool Use to Site Fuction. Use-wear analysis in some Final Upper Palaeoliythic sites in the Basque country. B.A.R. International Series 658. Oxford.

Ibáñez Estévez, J. J., González Urquijo, J. E., Ruiz Idarraga, R., Berganza Gochi, E., 1993. Huellas de uso en sílex en el yacimiento de Santa Catalina. Consideraciones sobre la manufactura del utillaje óseo y la funcionalidad del asentamiento. En: Anderson, P. C., Beyries, S., Otte, M., Plisson, H. (Dirs), Traces et function: les gestes retrouvés, 225-234. Liège.

Laborda, Martínez, M. A., 2013. Reconstrucción funcional de cuatro cantos rodados con estigmas de uso de la cueva de Zatoya (Navarra). Munibe Antropologia-Arkeologia 64, 43-68.

Lefort, J. P., 2014, Deux apports pleistocenes exotiques: le basalte et la pierre ponce. quelques considerations concernant leurs origines. Journée du "CReAAH". Archéologíe, Archéosciences, Historire, 1-2. Centre de Recherche de Archéologíe, Archéosciences, Historire, Rennes.

Mujika, J. A., 1983. Industria de hueso en la Prehistoria de Guipúzcoa. Munibe 35(3-4), 451-631.

Passemard, E., 1944. La caverne d’Isturitz en pays Basque. La Préhistoire 9, 7-95.

Pétillon, J. M., 2013. Circulation of whale-bone artifacts in the northern Pyrenees during the late Upper Paleolithic. Journal of Human Evolution 65, 525-543.
Pétillon, J. M., 2016. Vivre au bord du golfe de Gascogne au Paléolithique supérieur récent: vers un nouveau paradigme. En: Dupont, C., Marchand, G. (Dirs.), Archéologie des chasseurs-cueilleurs maritimes. De la fonction des habitats à l'organisation de l'espace litoral. Actes de la Séance de la Société préhistorique française, Rennes, France, abril 2014, 23-36.

Pétillon, J. M., Cattelain, P., Normand, C., Schwab, C., Szmid, C., 2009. Premières dates radiocarbone pour le Magdalénien d'Isturitz (Pyrénées-Atlantiques). Bulletin de la Société préhistorique française 106(3), 583-601.

Piette, E., 1873. Sur la grotte de Gourdan, sur la lacune que plusieurs auteurs placent entre l'âge du renne et celui de la Pierre polie, et sur lárt paléolithique dans ses rapports avec I'art gaulois. Bulletin de la Société d'anthropologie de Paris $1 \mathrm{I}^{\circ}$ Série t.8, 384-425.

Rivero Vilá, O., 2010. La movilidad de los grupos humanos en el Magdaleniense de la Región Cantábrica y los Pirineos: una visión a través del arte. Universidad de Salamanca.

Roselló-Izquierdo, E., Morales-Muñiz, A., 2014. Las ictiofaunas de Santa Catalina (Lequeitio, Vizcaya): un registro singular para la prehistoria cantábrica. En: Berganza, E., Arribas, J. L. (coords.), La cueva de Santa Catalina (Lekeitio, Bizkaia): La intervención arqueológica. Restos vegetales, animales y humanos. Kobie, serie BAI 4, 161-262. Diputación Foral de BizkaiaBizkaiko Foru Aldundia, Bilbao.

Roselló-Izquierdo, E., Berganza-Gochi, E., Nores-Quesada, C., Morales-Muñiz, A., 2015. Santa Catalina (Lequeitio, Basque Country): An ecological and cultural insight into the nature of prehistoric fishing in Cantabrian Spain. Journal of Archaeological Science: Reports. https://doi.org/10.1016/j.jasrep.2015.06.002

Tarriño Vinagre, A., Elorrieta Baigorri, I., García Rojas, M., 2015. Flint as raw material in prehistoric times: Cantabrian Mountain and Western Pyrenees data. Quaternary International 364, 94-108.

Tarriño Vinagre, A., Muñoz-Fernández, E., Elorrieta Baigorri, I., Normand, C., Rasines del Río, P., García Rojas, M., Pérez-Bartolomé, M., 2016. El sílex en la Cuenca Vasco-cantábrica y el Pirineo occidental: materia prima lítica en la Prehistoria. CPAG 26, 191-228.

Vásquez Sánchez, V. F.; Rosales Tham, T. E., 2014. Malacofauna de la cueva de Santa Catalina". En: Berganza, E., Arribas, J. L. (coords.), La cueva de Santa Catalina (Lekeitio, Bizkaia): La intervención arqueológica. Restos vegetales, animales y humanos. Kobie, serie BAI 4, 119-150. Diputación Foral de BizkaiaBizkaiko Foru Aldundia, Bilbao. 